\title{
Modeling and Analysis in Support of Decision Making for Technological Investment
}

April 11, 2003

Prepared by

S. Lenhart

V. Protopopescu 


\title{
DOCUMENT AVAILABILITY
}

Reports produced after January 1, 1996, are generally available free via the U.S. Department of Energy (DOE) Information Bridge:

Web site: http://www.osti.gov/bridge

Reports produced before January 1,1996, may be purchased by members of the public from the following source:

\author{
National Technical Information Service \\ 5285 Port Royal Road \\ Springfield, VA 22161 \\ Telephone: 703-605-6000 (1-800-553-6847) \\ TDD: 703-487-4639 \\ Fax: 703-605-6900 \\ E-mail: info@ntis.fedworld.gov \\ Web site: http://www.ntis.gov/support/ordernowabout.htm
}

Reports are available to DOE employees, DOE contractors, Energy Technology Data Exchange (ETDE) representatives, and International Nuclear Information System (INIS) representatives from the following source:

Office of Scientific and Technical Information

P.O. Box 62

Oak Ridge, TN 37831

Telephone: $865-576-8401$

Fax: 865-576-5728

E-mail: reports@adonis.osti.gov

Web site: http://www.osti.gov/contact.html

\begin{abstract}
This report was prepared as an account of work sponsored by an agency of the United States Government. Neither the United States government nor any agency thereof, nor any of their employees, makes any warranty, express or implied, or assumes any legal liability or responsibility for the accuracy, completeness, or usefulness of any information, apparatus, product, or process disclosed, or represents that its use would not infringe privately owned rights. Reference herein to any specific commercial product, process, or service by trade name, trademark, manufacturer, or otherwise, does not necessarily constitute or imply its endorsement, recommendation, or favoring by the United States Government or any agency thereof. The views and opinions of authors expressed herein do not necessarily state or reflect those of the United States Government or any agency thereof.
\end{abstract}


ORNL-2003/116

\title{
MODELING AND ANALYSIS IN SUPPORT OF DECISION MAKING FOR
} OPTIMAL TECHNOLOGICAL INVESTMENT

\author{
S. Lenhart \\ V. Protopopescu
}

April 11, 2003

\author{
Prepared by \\ OAK RIDGE NATIONAL LABORATORY \\ P.O. Box 2008 \\ Oak Ridge, Tennessee 37831-6285 \\ managed by \\ UT-Battelle, LLC \\ for the \\ U.S. DEPARTMENT OF ENERGY \\ under contract DE-AC05-00OR22725
}





\section{CONTENTS}

Page

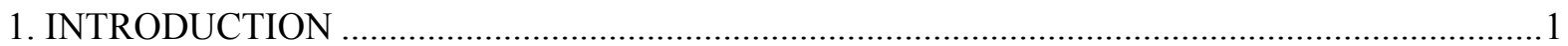

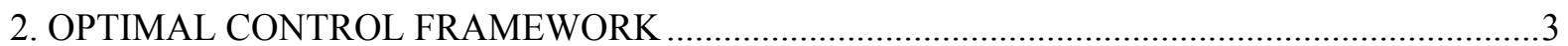

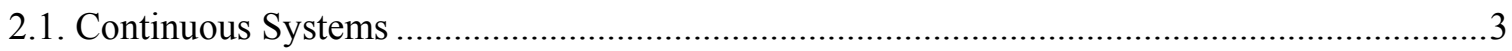

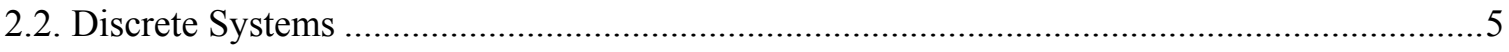

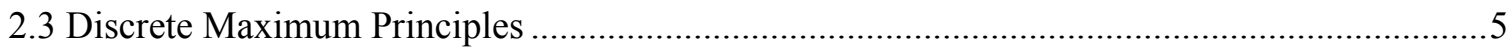

3. SENSITIVITY OF THE OPTIMAL CONTROL TO VARIOUS FACTORS ................................

4. OPTIMAL CONTROL FORMULATION FOR CONTINUOUS SYSTEMS WITH DISCRETE

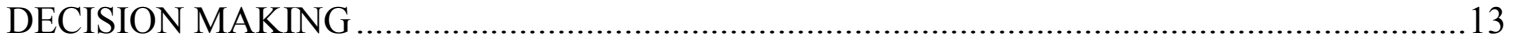

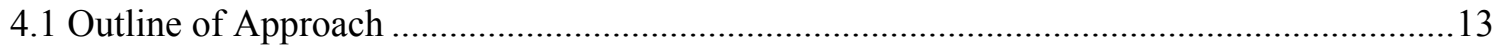

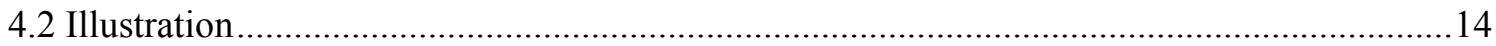

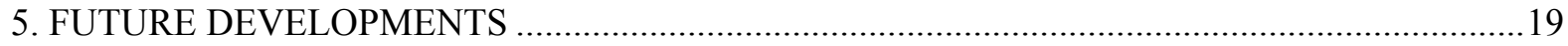

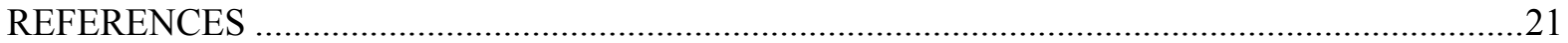




\section{INTRODUCTION}

Engineering design, resource allocation, military operations, and investment strategies share a major common trait, which is, to a large extent, independent of their different origins, specific features, and intended goals. The unifying trait is the fact that, in any of these endeavors, one has to make reasonable choices, at multiple levels of decision making, among various possible and sometimes competing prospective solutions to an important and consequential practical problem. While the specifics of the problem depend on application, context, additional constraints, etc., the ultimate - albeit imprecise goal in all these activities is to "optimize performance," which is to have maximal success/profit/return with minimal time/effort/investment.

In general, the underlying system is ruled by complex and often unknown dynamics, and affected by various uncertainties, which are unknown as well; on the other hand, there are numerous levels of decision making, which result in a hierarchical structure in the decision process (tree) that is both asynchronous and non-deterministic. Usually, indifferent of the specific application, as one lowers the level of decision making, alternatives depend on fewer independent variables and models become more detailed and physics/engineering based. On the contrary, at higher levels, various components aggregate and decision making is based more on fuzzier criteria instead of readily quantifiable physics/engineering details. Moreover, decisions are strongly influenced by the educational and personal biases of the people who take them. In some instances, this may blur, if not totally obfuscate objective comparisons between various options.

Therefore, a crucial point in decision-making is properly understanding and quantifying the tradeoffs, including all their future relevant consequences. Since the interaction between various choices is an intricate nonlinear process, the focus shifts from the dynamics itself to the overall performance and affordability. This is not unreasonable, since oftentimes major upgrades on some components have little impact, while minor upgrades of other components turn out to be critical.

To illustrate the approach, we assume that one deals with only two levels. At the lower level, physical/engineering processes are described by continuous and/or discrete, analytic and/or computer models. These models are supposed to be deterministic (e.g. dynamics as ruled by well established physical laws), but their outcome may depend in an unpredictable way on: (i) small nonlinearities unaccounted for in the model development and/or (ii) factors that - at the specific level of modelization may be treated as stochastic terms (weather conditions, human factors, political circumstances, fluctuations in the quality of carburant, wear and tear, etc.) To this extent, the outcomes of the model processes may be considered stochastic variables/fields with a certain probability distribution function (PDF). Upon many realizations of the model, one can get reliable information about the essential features of this PDF.

At the higher level, the process/mission can be viewed as comprised of a finite number of discrete nodes, which represent the major articulations of the mission, as well as its crucial decision points. Based upon these decisions, one may alter the course of a particular mission, the plan for future missions, future investments in science and development, etc. The links between the nodes are realized by "black boxes." At the lower level, these black boxes contain the physical/engineering models of various parts/components of the mission. The ultimate goal - as already mentioned - is to improve decision making (i.e., to reduce the use of resources and to maximize the expected result) by better taking advantage of the information available at the lower level. Setting this requirement into a mathematically well posed and hopefully tractable problem depends crucially on the selection of a suitable objective functional (OF), which properly quantifies "effort" and "success." The difficulty in choosing an appropriate OF stems from the fact that, at a fundamental level, "assigning value" to an object, action, or feature is not a purely logical, but an axiologic process, whereby subjective determinations are weighed against each other. Thus, there is no "objective," systematic procedure to organize values within a uniquely defined well ordered set. 
A brief review of the literature ${ }^{12,13,14}$ indicates that usually researchers approach decision making in complex systems at the lower levels, namely by using physics or engineering based models. In the following, we shall show that one can base rational and perhaps more efficient decision making on system performance at a given level, rather than physics and/or engineering based considerations. Indeed, as mentioned before, when dealing with very complex systems, one lacks detailed comprehensive knowledge about their structure and dynamics, so system performance constrained by affordability considerations plays a primary role in decision making.

The framework above leads naturally to a multi-criteria optimization problem and looking for the optimal solution under the set of existing constraints. In general, the analysis should include a probabilistic component, dealing with risk and various sources of uncertainty. This optimization problem can be solved by an optimization algorithm, or by optimal control (OC) methods. For reasons that will become obvious during the analysis, we believe that OC offers the suitable solution. OC theory is a branch of applied mathematics developed to find "best ways" to steer a dynamical system to achieve a desired goal. Assuming a deterministic dynamics, the evolution of the dynamical system may be continuous in time, discrete, or hybrid, while the other independent variables may be continuous or discrete as well. The maximum principle for optimal control of ordinary differential equations was developed in the late ' 50 's by L. S. Pontryagin and his co-workers ${ }^{3}$. Shortly thereafter the principle was generalized to discrete time systems ${ }^{2}$. While generalization to distributed systems, described by partial differential equations, is not automatic, a general framework was developed during the ' 60 's by J.-L. Lions and co-workers in which OC problems can be properly set and their solution be systematically attempted $^{4,10}$.

For an application where the dynamics is incompletely known or unavailable, one has to resort to a stochastic description, whereby the details of the unknown dynamics at the lower level are accounted for in a PDF that contains all the available information about the system. The PDF is then used at the higher level in the OC approach as developed for stochastic evolution equations ${ }^{11}$.

To illustrate our approach, we shall limit the analysis to a deterministic framework where - to a certain extent - the effects of uncertainty can be suitably modeled by varying the balance between price and reward. We then extend Pontryagin's principle to hybrid systems, for which variables may change continuously and discretely. This framework is the most relevant for the decision making process, where the lower level dynamics can be discrete or continuous while the decision making process usually takes place discontinuously, at discrete times. De-scoping can also be included in a natural manner by discounting the $\mathrm{OF}$ as time evolves. 


\section{OPTIMAL CONTROL FRAMEWORK}

The behavior of the underlying dynamical system is described by a state that belongs to a certain functional space. We assume that there is a way to control the state by acting upon it with a suitable control. Thus the (dynamics of the) state, $x$, depends on the control, $u$. The possible types of evolution of the state system include:

(i) continuous time evolution

$$
x^{\prime}=g(t, x, u)
$$

(ii) discrete time evolution

$$
x_{n+1}=x_{n}+g\left(u_{n}, x_{n}, n\right)
$$

or (iii) a combination thereof.

The goal is to adjust control $u$ to maximize a given objective functional, $J(u(t), x(t), t)$, that balances judiciously the desired goal and the required cost needed to reach it. The optimal solution is obtained when the "most" goal is obtained with "least" cost. In general, the functional $J$ depends on the control and the state. Note that since the state $x$ depends on the control, $u$, the functional $J$ depends in fact only on the control $u$. In the following, we shall briefly review some of the main results of optimal control theory for continuous and discrete systems ${ }^{3}$.

\subsection{CONTINUOUS-TIME SYSTEMS}

For the continuous time case, when evolution is described by ordinary differential equations, the OC problem is solved by using Pontryagin's Maximum Principle.

Basic ODE Optimal Control Problem. Find a piecewise continuous control, $u(t)$, and its associated state variable, $x(t)$, which maximizes the objective functional

$$
J(u)=\int_{0}^{T} f(t, x(t), u(t)) d t
$$

subject to

$$
\begin{aligned}
& x^{\prime}(t)=g(t, x(t), u(t)) \\
& x(0)=x_{0} \\
& x(T) \text { - not specified }
\end{aligned}
$$


over the set of all admissible controls. Note that minimization problems can be handled easily, since $-\min _{u} J(u)=\max _{u}(-J(u))$. In the following, we shall consider extremal problems of both types.

Pontryagin's Maximum Principle. This principle converts the maximization of the "integral" objective functional, $J$, coupled with the state system described by a set of ordinary differential equations into maximizing pointwise the Hamiltonian with respect to the control. We define the Hamiltonian

$$
H(t, x(t), u(t), \lambda(t))=f(t, x, u)+\lambda(t) g(t, x, u)
$$

Pontryagin's Maximum Principle states that if $u^{*}(t), x^{*}(t)$ are optimal, then there exists an adjoint variable $\lambda(t)$ such that

$$
H\left(t, x^{*}, u, \lambda\right) \leq H\left(t, x^{*}, u^{*}, \lambda\right), \text { for all } t \in[0, T] .
$$

Further, $\lambda^{\prime}(t)=-H_{x}\left(t, x^{*}, u^{*}, \lambda\right), \quad \lambda(T)=0$.

If the Hamiltonian is differentiable with respect to the control, one has also the optimality condition

$$
H_{u}\left(t, x^{*}, u^{*}, \lambda\right)=0
$$

There are situations in which this condition is not satisfied (e.g., bang-bang controls, see below). Note the concavity or convexity of $H$ with respect to the control determines whether a maximum or minimum of the objective functional is attained.

Bounded Controls. Suppose the practical situation imposes upper and lower bounds on the controls:

$$
a \leq u(t) \leq b \text { for all } t
$$

If the Hamiltonian is linear in $u$,

$$
H=h\left(t, x^{*}, \lambda\right) u+k\left(t, x^{*}, \lambda\right):
$$

then $u^{*}$ maximizes $H$ with respect to $u \Rightarrow$

If $h\left(t, x^{*}(t), \lambda(t)\right)>0$, then $u^{*}(t)=b$

If $h(t, x *(t), \lambda(t))<0$, then $u *(t)=a$ 
If $h\left(t, x^{*}(t), \lambda(t)\right)=0$ for an interval of time, then $u *(t)$ is "singular."

Since we are choosing $u^{*}$ to maximize $H$ and the coefficient of $u^{*}$ vanishes on a time interval (singular case), other features of the problem must be used to determine $u^{*}$.

Controls that only take values at the extremes, $a$ or $b$, are called "bang-bang."

If the singular case does not occur, then a Hamiltonian linear in $u$ will lead to "bang-bang" controls. This result is not surprising, since the maximum of a linear function with non-zero slope on a closed bounded interval occurs at one of the endpoints of the interval. Here the slope $h\left(t, x^{*}, \lambda\right)$ of the linear function in $u$ may be changing with time.

\subsection{DISCRETE-TIME SYSTEMS}

In the discrete time case the control and state variables have the form:

control $u=\left(u_{o}, u_{1}, \ldots, u_{N-1}\right)$

state $y=\left(y_{0}, y_{1}, \ldots, y_{N}\right)$

$$
y_{n+1}=y_{n}+f\left(y_{n}, u_{n}, n\right), \quad n=0,1, \ldots, N-1
$$

with $y_{o}$ given. Once again, we note that the state $y$ is dependent on the control input, $u$.

We adjust control to maximize a given functional:

$$
J(u)=\sum_{n=0}^{N-1} F\left(y_{n}, u_{n}, n\right)+S\left(y_{N}\right)
$$

The problem is to find $u^{*}$ such that

$$
J\left(u^{*}\right)=\max _{u} J(u)
$$

We denote by $y^{*}$ the corresponding optimal state.

\subsection{DISCRETE MAXIMUM PRINCIPLE}

Pontryagin's Maximum Principle can be generalized to include discrete evolutions ${ }^{2}$. For each $n=1,2, \ldots, N-1$, define the Hamiltonian 


$$
H_{n}=H\left(y_{n}, u_{n}, \lambda_{n+1}, n\right)=F\left(y_{n}, u_{n}, n\right)+\lambda_{n+1} f\left(y_{n}, u_{n}, n\right)
$$

If $u_{n}^{*}, y_{n}^{*}$ are optimal, then there exists an adjoint variable, $\lambda_{n}$, such that

$$
\begin{aligned}
& \lambda=\left(\lambda_{0}, \lambda_{1}, \ldots, \lambda_{N}\right) \\
& \lambda_{n-1}=\lambda_{n}+\frac{\partial H_{n-1}}{\partial y_{n-1}} \quad n=0,1, \ldots, N-1 \\
& \lambda_{N}=\frac{\partial S\left(y_{N}\right)}{\partial y_{N}} \\
& H\left(y_{n}^{*}, u_{n}, \lambda_{n+1}, n\right) \leq H\left(y_{n}^{*}, u_{n}^{*}, \lambda_{n+1}, n\right)
\end{aligned}
$$

\section{Example 2.3}

Given the control $u$ and state $y$ with a component at each time interval, we consider the following optimization problem ${ }^{2}$ :

$$
\begin{aligned}
& y_{n+1}=y_{n}+u_{n}-d_{n} \\
& y_{0} \text { given } \quad n=0,1, \ldots, N-1 \\
& u_{n} \geq 0 \\
& \max _{u}\left(-\sum_{n=0}^{N-1} \frac{1}{2}\left[h\left(y_{n}-\hat{y}\right)^{2}+c\left(u_{n}-\widehat{u}\right)^{2}\right]\right)
\end{aligned}
$$

Given the Hamiltonian

$$
H_{n}=\frac{-1}{2}\left[h\left(y_{n}-\widehat{y}\right)^{2}+c\left(u_{n}-\widehat{u}\right)^{2}\right]+\lambda_{n+1}\left(u_{n}-d_{n}\right)
$$

we can find the dynamics of the adjoint variable:

$$
\begin{aligned}
& \lambda_{n-1}=\lambda_{n}-h\left(y_{n-1}-\hat{y}\right) \quad n=1, \ldots, N \\
& \lambda_{N}=0
\end{aligned}
$$

Since $H_{n}$ is maximized with respect to $u_{n}$ at $u_{n}^{*}$, we have 


$$
\frac{\partial H_{n}}{\partial u_{n}}=-c\left(u_{n}-\widehat{u}\right)+\lambda_{n+1}=0 \text { at } u_{n}^{*}
$$

subject to the constraints $u_{n}^{*} \geq 0$. Solving for the optimal control, we obtain:

$$
u_{n}^{*}=\max \left(0, \hat{u}+\frac{\lambda_{n+1}}{c}\right) .
$$

The above characterization of $u_{n}^{*}$ together with state and adjoint equations comprises the optimality system. 


\section{SENSITIVITY OF THE OPTIMAL CONTROL TO VARIOUS FACTORS}

In this section we give a few examples that show the extreme sensitivity of the OC to various factors that enter the definition of the objective functional. These examples show that the judicious choice of the objective functional is paramount to the correct solution of the decision making problem. While the OC approach is canonical and not subject to change, the form of the objective functional depends on subjective and variable appreciations and should be considered very carefully.

The following examples illustrate the strong dependence of nature and characterization of solutions to OC problems on: (i) length of the time interval over which the problem is set; (ii) presence or absence of control constraints; and (iii) magnitude of the constraints.

\section{Example 3.1}

Changing the time interval from $[0,1]$ to $[0,2]$ results in a different characterization of the OC on the common interval.
a)

$$
\text { Find } \max _{u} \int_{0}^{1}\left(2 x-3 u-u^{2}\right) d t
$$

under the conditions

$$
(*)\left[\begin{array}{l}
x^{\prime}=x+u \\
x(0)=5 \\
0 \leq u(t) \leq 2
\end{array}\right.
$$

Forming the Hamiltonian, $H=2 x-3 u-u^{2}+\lambda(x+u)$, we use $\frac{\partial H}{\partial u}=0$ at $u^{*}$ and the adjoint system $\lambda^{\prime}=-2-\lambda, \lambda(1)=0$, to find the optimal control. The optimal control is

$$
u_{1} *(t)=\left[\begin{array}{cc}
e^{1-t}-2.5 & \text { if } 0 \leq t \leq 1-\ln 2.5 \\
0 & \text { if } t>1-\ln 2.5
\end{array}\right.
$$

b) Find $\max _{u} \int_{0}^{2}\left(2 x-3 u-u^{2}\right) d t$ under the same conditions $\left(^{*}\right)$. Now the optimal control is

$$
u_{2}^{*}(t)=\left[\begin{array}{ll}
2 & \text { if } 0 \leq t \leq 2-\ln 4.5 \\
e^{2-t}-2.5 & \text { if } 2-\ln 4.5<t \leq 2-\ln 2.5 \\
0 & \text { if } t>2-\ln 2.5
\end{array}\right.
$$


and the two optimal controls do not agree on $0 \leq t \leq 1$.

\section{Example 3.2}

The nature of control changes drastically with respect to a small change in final time.

a) $\min _{u} \int_{0}^{1} x^{2} d x$

$$
\text { (*) }\left[\begin{array}{l}
x^{\prime}=u \\
x(0)=1 \\
-1 \leq u(t) \leq 1
\end{array}\right.
$$

We want to minimize the area under the $x^{2}$ graph. Since $\mathrm{x}(0)=1$, we want to decrease $\mathrm{x}$ as rapidly as possible. The optimal control is $u_{1}^{*}(t)=-1$ on $0 \leq t \leq 1$.

b) $\quad \min _{u} \int_{0}^{1+\delta} x^{2} d x$ with $\delta>0$ and the conditions $(*)$ above.

Once $x^{*}$ reaches 0 , we choose $u^{*}$ to keep $x^{*}$ at 0 for the remaining time. The optimal control is

$$
u_{2}^{*}(t)=\left[\begin{array}{lll}
-1 & \text { on } & 0 \leq t<1 \\
0 & \text { on } & 1 \leq t \leq 1+\delta
\end{array}\right.
$$

Note that the Hamiltonian is $H=x^{2}+\lambda u$.

On $1 \leq t \leq 1+\delta, \lambda(t) \equiv 0$, which means that, on the specified interval, the control term simply drops out of $H$. To minimize the Hamiltonian, we choose $u^{*}$ to minimize $\left(x^{*}\right)^{2}$ yielding $u^{*} \equiv 0$ on the final part of the time interval. Thus the change of the final time from 1 to $1+\delta$, alters the nature of the control from regular to singular.

\section{Example 3.3}

The following example illustrates how the presence of control constraints alters significantly the optimal control ${ }^{1}$.

$$
\begin{gathered}
\min _{u} \int_{0}^{1}\left(u^{2}+x\right) d t \\
x^{\prime}=u \\
x(0)=0 \\
x(1)=\frac{1}{16}
\end{gathered}
$$


a) The solution without a control constraint gives

optimal control, $u_{1}^{*}(t)=\frac{1}{2}\left(t-\frac{3}{8}\right)$

optimal state, $x_{1}^{*}(t)=\frac{t^{2}}{4}-\frac{3 t}{16}$

Note the adjoint equation, $\lambda^{\prime}=-1$, does not have a final boundary condition, since the state, $x$, has two boundary conditions.

b) The solution with control constraint, $u(t) \geq 0$, gives

$$
\begin{aligned}
& \text { optimal control } u_{2}^{*}(t)=\left[\begin{array}{l}
0,0 \leq t \leq \frac{1}{2} \\
\frac{1}{2}\left(t-\frac{1}{2}\right), \quad \frac{1}{2} \leq t \leq 1
\end{array}\right. \\
& \text { optimal state } x_{2}^{*}(t)=\left[\begin{array}{l}
0,0 \leq t \leq \frac{1}{2} \\
\frac{1}{4}\left(t-\frac{1}{2}\right)^{2},
\end{array} \quad \frac{1}{2} \leq t \leq 1\right.
\end{aligned}
$$

The truncation of the negative part of the optimal control in part a) does not give the optimal control in part b). The two boundary conditions on the state make this problem unusual. The constraint must be an intrinsic part of solution.

\section{Example 3.4}

There is a strong dependence of the optimal control on the upper bound on the control set.

a) $\max _{u} \int_{0}^{1}(x-u) d t$

$$
\begin{aligned}
& x^{\prime}=2 u(1-u) \\
& 0 \leq u(t) \leq 1 \\
& x(0)=0
\end{aligned}
$$

From the adjoint system, $\lambda^{\prime}=-1, \lambda(1)=0$, the adjoint variable is $\lambda(t)=1-t$, and the optimal control satisfies $u^{*}=\frac{1}{2}-\frac{1}{4 \lambda}$, subject to the constraints. The optimal control reads: 


$$
u_{1}^{*}(t)=\left[\begin{array}{ll}
\frac{1}{2}-\frac{1}{4(1-t)} & \text { if } 0 \leq t \leq \frac{1}{2} \\
0 & \text { if } t \geq \frac{1}{2}
\end{array}\right.
$$

b) same as above except $0 \leq u(t) \leq \frac{1}{8}$

The optimal control is:

$$
u_{2}^{*}(t)=\left[\begin{array}{ll}
\frac{1}{8} & \text { if } 0 \leq t \leq \frac{1}{3} \\
\frac{1}{2}-\frac{1}{4(1-t)} & \text { if } \frac{1}{3} \leq t \leq \frac{1}{2} \\
0 & \text { if } t \geq \frac{1}{2}
\end{array}\right.
$$




\section{OPTIMAL CONTROL FORMULATION FOR CONTINUOUS SYSTEMS WITH DISCRETE DECISION MAKING}

We consider examples which are discrete in time and continuous in other variables. Since Pontryagin's Maximum Principle is not known for such hybrid systems, we derive optimality conditions on a case by case basis, starting from the particular system under consideration and using techniques from the discrete maximum principle ${ }^{2}$ and the OC theory for partial differential equations ${ }^{4}$. The results contained in this section represent an original contribution to the general theory of OC in hybrid systems.

\subsection{OUTLINE OF APPROACH}

The control and state variables are discrete in time and continuous in the ( $n$-dimensional) $x$ variable. The $x$-variable represents all the physical variables of the problem, including physical time, which is continuous, and is not to be confused with the decision making time variable, $n$, which is discrete.

We introduce the vectors $u(x)=\left(u_{0}(x), u_{1}(x), \ldots, u_{N-1}(x)\right)$ and $y(x)=\left(y_{0}(x), y_{1}(x), \ldots, y_{N}(x)\right)$, that denote the control and state, respectively.

The state system reads

$$
\begin{gathered}
y_{n+1}(x)=A\left(y_{n}, u_{n}, x, n\right) \\
n=0,1, \ldots, N-1 \\
x \in \Omega \subset \mathfrak{R}^{m} \\
y_{0} \text { given }
\end{gathered}
$$

and the objective functional is given by

$$
J(u)=\sum_{n=0}^{N-1} \int_{\Omega} F\left(y_{n}(x), u_{n}(x), x, n\right) d x+\int_{\Omega} S\left(y_{N}(x)\right) d x
$$

The optimization problem is to find $u^{*}$ such that $J\left(u^{*}\right)=\max _{u} J(u)$.

(Note that boundary conditions on $y$ in the $x$ variables may be needed, depending on the nature of the operator $A$.)

To derive the characterization of $u^{*}$, we differentiate component-wise the map $u \rightarrow J(u)$. This operation requires the differentiation of the control-to-state map $u \rightarrow y(u)$. The sensitivity variables are directional derivatives of the map $u \rightarrow y(u)$ :

$$
\frac{y(u+\varepsilon \ell)-y(u)}{\varepsilon} \rightarrow \psi, \text { as } \varepsilon \rightarrow 0
$$


Note $\psi$ may depend on $u, y, \ell$, where $\ell$ is the variation direction. The sensitivity vector $\psi$ satisfies the system

$$
\psi_{n+1}=L\left(\psi_{n}, u_{n}, \ell_{n}, y_{n}, x, n\right)
$$

where $L$ is a linear operator in $\psi_{n}$, but may depend nonlinearly on other variables. We introduce the adjoint variables

$$
\begin{aligned}
& \lambda_{n-1}=L^{*}\left(\lambda_{n}, u_{n-1}, \ell_{n-1}, y_{n-1}, x\right)+\frac{\partial F\left(y_{n-1}, u_{n-1}, x, n-1\right)}{\partial y_{n-1}} \\
& \quad n=N, \ldots, 1 \\
& \lambda_{N}=\frac{\partial S\left(y_{N}(x)\right)}{\partial y_{N}}
\end{aligned}
$$

where $L^{*}$ is the adjoint of the operator $L$. Using $\lambda$ and $\psi$ in simplifying the directional derivative of $J$ :

$$
0 \leq \lim _{\varepsilon \rightarrow 0^{+}} \frac{J\left(u^{*}+\varepsilon \ell\right)-J\left(u^{*}\right)}{\varepsilon},
$$

we obtain the characterization of the optimal control in the form

$$
u^{*}=G\left(\lambda^{*}, y^{*}\right)
$$

where $G$ is, in principle, an explicitly known function. The optimality system consists of the state and adjoint equations together with the above characterization.

\subsection{ILLUSTRATION}

\section{Example 4.2.1}

Given control $u=\left(u_{0}(x), \ldots, u_{N-1}(x)\right)$, the corresponding state system is given by 


$$
\begin{aligned}
& y=\left(y_{0}(x), y_{1}(x), \ldots, y_{N}(x)\right), \quad x \in \Omega \subset \mathfrak{R}^{1} \\
& y_{n+1}(x)=A\left(y_{n}(x), u_{n}(x)\right)=u_{n}(x) y_{n}(x)+\frac{\partial y_{n}}{\partial x}(x) \quad \text { at time steps } n=0,1,2, \ldots, N-1 \\
& y_{n}(x)=0 \text { for } x \in \partial \Omega, \quad n=0,1,2, \ldots, N \\
& y_{0}(x) \text { given. }
\end{aligned}
$$

We seek to find $\min _{u} J(u)$, i.e. to minimize the functional

$$
J(u)=\int_{\Omega}\left[y_{N}(x)+\frac{1}{2} \sum_{n=0}^{N-1}\left(u_{n}(x)\right)^{2}\right] d x .
$$

The adjoint variables

$$
\begin{aligned}
& \lambda=\left(\lambda_{0}(x), \lambda_{1},(x), \ldots, \lambda_{N}(x)\right) \\
& \lambda_{N}(x)=1
\end{aligned}
$$

satisfy the equations

$$
\begin{array}{ll}
\lambda_{n-1}(x)=\lambda_{n}(x) u_{n-1}^{*}(x)-\frac{\partial \lambda_{n}(x)}{\partial x}, & n=N, \ldots . ., 1 \\
\lambda_{n}(x)=0 \text { for } x \in \partial \Omega, & n=N-1, \ldots .1,0
\end{array}
$$

By differentiating the maps, $u \rightarrow J(u)$ and $u \rightarrow y(u)$, with respect to $u$ at $u *$, and using the adjoint equations, we obtain

$$
u_{n}^{*}(x)=-\lambda_{n+1}(x) y_{n}^{*}(x), \quad n=0,1, \ldots, N-1
$$

The optimal control is represented in terms of the optimality system, the state and adjoint quotations combined with characterization $(*)$. 


\section{Example 4.2.2}

The state system reads

$$
\begin{aligned}
& y_{n+1}(x)=A\left(y_{n}(x), u_{n}(x)\right)=\left(1-u_{n}(x)\right) \int_{\Omega} k(x, s) r y_{n}(s) d s, \quad n=0,1, \ldots, N-1 \\
& \text { with control } u=\left(u_{o}(x), u_{1}(x), \ldots, u_{N-1}(x)\right), \quad x \in \Omega \subset \mathfrak{R}^{1} .
\end{aligned}
$$

The objective functional is

$$
J(u)=\sum_{n=0}^{N-1} \int_{\Omega}\left[A_{n} u_{n}(x) \int_{\Omega} k(x, s) r y_{n}(s) d s-\frac{\beta}{2}\left(u_{n}\right)^{2}(x)\right] d x
$$

which is to be maximized over the set of admissible controls. The adjoint system is

$$
\begin{aligned}
& \lambda_{N}(x)=0 \\
& \lambda_{n-1}(x)=\int_{\Omega} k(s, x) r\left(1-u_{n-1}(s)\right) \lambda_{n}(s) d s+A_{n-1} \int_{\Omega} k(s, x) r u_{n-1}(s) d s
\end{aligned}
$$

The characterization of optimal control is

$$
u_{n}^{*}=\frac{1}{\beta}\left(A_{n}-\lambda_{n+1}\right) \int_{\Omega} k(x, s) r y_{n}(s) d s, \quad n=0,1, \ldots N-1
$$

The optimality system is the state and adjoint equations together with the above characterization.

\section{Example 4.2.3}

The two examples before were given for illustration purposes only, as simple instances in which discrete and continuous variables are mixed. The continuous variables did not explicitly contain time and only boundary conditions had to be imposed on the operator $A$. The next example illustrates a much more realistic situation, whereby the continuous evolution takes place in both time and space. Upon this continuous evolution, a discrete decision making process is superimposed, at times $0, T, 2 T, \ldots .,(N-1) T$. The time intervals for the continuous evolution and the decision process do not have to be equal.

State: $\quad y=\left(y_{0}(x, t), y_{1}(x, t), \ldots, y_{N}(x, t)\right)$

Control: $\quad u=\left(u_{0}(x, t), \ldots, u_{N-1}(x, t)\right)$ 
For spatial variables $x$ and time variable $t,(x, t) \in Q=\Omega \times(0, T), \Omega \subset \mathfrak{R}^{m}$, and decision time step $n$, we consider the state system:

$$
\frac{\partial y_{n+1}}{\partial t}=\Delta y_{n+1}+u_{n} y_{n} \text { in } Q
$$

where $\Delta$ denotes the Laplacian in the space variables.

The initial and boundary conditions are:

$$
\begin{array}{ll}
y_{n+1}(x, 0) & =y_{n+1,0}(x) \\
y_{n+1}(x, t) & =0
\end{array} \quad \text { on } \partial \Omega \times(0, T)
$$

We assume that the starting state, $y_{0}(x, t)$, is known. The goal is to maximize the objective functional

$$
J(u)=\int_{\Omega} y_{N}(x, T) d x-\sum_{n=0}^{N-1} \int_{Q} \frac{\beta_{n}}{2} u_{n}^{2}(x, t) d x d t
$$

over the available control space.

The adjoint vector function, $\lambda=\left(\lambda_{0}(x, t), \lambda_{1}(x, t), \ldots, \lambda_{N}(x, t)\right)$, satisfies the system

$$
\frac{-\partial \lambda_{n}}{\partial t}=\Delta \lambda_{n}+u_{n} \lambda_{n+1} \quad \text { in } Q, \quad n=0,1, \ldots, N-1
$$

supplemented with final and boundary conditions

$$
\begin{aligned}
& \lambda_{n}(x, T)=0 \quad n=0,1, \ldots, N-1 \\
& \lambda_{n}(x, t)=0 \quad \text { on } \partial \Omega \times(0, T), \quad n=0,1, \ldots, N-1
\end{aligned}
$$

and with the additional evolution equation

$$
\begin{array}{lr}
\frac{-\partial \lambda_{N}}{\partial t}-\Delta \lambda_{N}=0 & \text { in } Q \\
\lambda_{N}(x, T)=1, & x \in \Omega
\end{array}
$$




$$
\lambda_{N}(x, t)=0 \quad \text { on } \partial \Omega \times(0, T)
$$

for the last element of the adjoint vector, $\lambda_{N}$.

The characterization of the optimal control is $u_{n}^{*}=\frac{1}{\beta_{n}} y_{n} \lambda_{n+1}$. 


\section{FUTURE DEVELOPMENTS}

1. From the application standpoint, the first question to be addressed has to do with the correct, or at least reasonable, format of the objective functional. Indeed, in any optimization problem, one has to optimize something (in formal terms known as an objective functional). The specific form of the objective functional is of paramount importance in determining the "winning" strategy. The few examples in section 3 illustrate sufficiently the extreme sensitivity of the result with respect to (various elements of) the objective functional. Once a reasonable objective functional is agreed upon, we will apply the approach developed here and determine the best course of action, as given by the OC theory. We think that this should be the natural next phase of this effort.

2. From the mathematical standpoint, the new results on hybrid systems, as outlined in Section 4, will have to be developed and justified rigorously. Existence, uniqueness, and characterization of the optimal control and the format of the adjoint system must be established for specific systems and objective functionals. Issues of compactness of maximizing sequences of controls and corresponding weak convergence results are crucial. Indeed, objective functionals and state systems do not always depend continuously on sequences of controls with weak convergence properties. The theory, justification, and corresponding application of OC for such hybrid systems would be an original contribution in applied mathematics, optimal control theory, and decision making theory. Moreover, most OC results for standard systems are derived under various types of convexity conditions. More general conditions to guarantee a solution of the OC problem must be developed for the functionals of interest, which may not be convex and may be non-standard. This work will have to be done either independently or in conjunction with the implementation of the theory on an objective functional that goes beyond a reasonable study model and has direct implications for NASA programs. In that case, the rigorous foundation of the approach developed in Section 4 becomes absolutely necessary.

3. Developing the numerical algorithm for the approach in Section 4 - even for a study problem requires the solution of a large nonlinear system of forward backward equations (two point boundary value problems). These problems are notoriously unstable, particularly for high dimensional systems. We have implemented iterative methods for numerical solutions of OC problems for both partial and ordinary differential equations. Starting with an initial guess for the OC, the state system is solved forward in time and then the adjoint system is solved backward in time. Using the updated state and adjoint values the control approximation is suitably updated and the forward and backward sweeps are completed again. The procedure continues until convergence of the iterates occurs. Solving OC problems for hybrid systems with discrete decision times introduces another level of difficulty, especially if the decision times are varying. Layers of iterations may have to be carried out at different time scales, namely the decision times and the characteristic times of the underlying system. Starting with a small number of fixed decision times would be the most logical place to start developing these new general algorithms.

4. After considering the case of a fixed set of decision times, one may consider varying this set, to allow for more realistic scenarios. One possible variation would be to fix the number of decisions and vary the times at which these decisions are made. Then one could tackle the even more difficult case with varying the number and timing of the decisions. The time schedule for the decisions is a new feature, which is very relevant to technological investment problems.

5. Considering the underlying state system as described by stochastic differential equations would turn the focus to stochastic OC. The theory of OC of stochastic differential equations is well developed, but including a discrete variable running at a different time scale would add a new and difficult feature. Another possible choice of underlying system is piecewise deterministic processes. These processes are ruled by deterministic dynamics, but jump to a different deterministic dynamics at random times. This scenario provides an alternate way to introduce stochasticity and uncertainty into the system. Our contention is that very complex NASA 
missions cannot be realistically treated within a probabilistic framework, since for key elements of the decision chain there are two few events to develop an empiric probabilistic understanding.

6. On the other hand, application of direct and/or adjoint sensitivity analysis (SA) and uncertainty analysis (UA) is warranted at least for portions of the decision chain and/or "what-if" simulation scenarios. The set of sensitivities are a crucial tool for assessing the importance of various parameters in the global dynamical outcome, while uncertainties and their time evolution (propagation) are necessary in dealing with unknown and/or uncertain conditions. Combined SA and UA ensure an approach whereby uncertainties and therefore risks are reduced.

7. While not formalizable within a probabilistic framework, decisions under very severe uncertainties may be addressed within the framework of the "information gap" theory, developed by Ben-Haim ${ }^{15}$. Info-gap theory opens a new avenue in the field of decision making insofar it is radically different from all current theories of decision under uncertainty. The difference originates in the modeling of uncertainty as an information gap rather than a probability.

8. Finally, one should address the quantification of the actual and potential "value" of a new discovery and factor it properly within the objective functional. This would touch upon the very difficult, but interesting question of introducing a proper order relation within the set of values. 


\section{REFERENCES}

1. M. I. Kamien and N. L. Schwartz, Dynamic Optimization, the Calculus of Variations and Optimal Control in Economics and Management, North Holland, New York, $2^{\text {nd }}$ edition, 1991.

2. S. P. Sethi and G. L Thompson, Optimal Control Theory, Applications to Management Science and Economics, Kluwer Publishers, Boston, $2^{\text {nd }}$ edition, 2000.

3. L. S. Pontryagin, V. G. Boltyanskii, R. V. Gamkrelidze, and E. F. Mischchenko, The Mathematical Theory of Optimal Processes, Wiley, New York, 1962.

4. X. Li and J. Yong, Optimal Control Theory for Infinite Dimensional Systems, Birkhauser Boston, 1995.

5. M. E. J. Newman, M. Girvan, and J. D. Farmer, "Optimal Design, Robustness and Risk Aversion," Phys. Rev. Lett., 89, 028301 (2002).

6. D. Challet and N. F. Johnson, "Optimal Combination of Imperfect Objects," Phys. Rev.Lett., 89, 028701 (2002).

7. J. M. R. Parrondo, G. P. Harmer, and D. Abbott, "New Paradoxical Games Based on Brownian Ratchets," Phys. Rev. Lett., 85, 5226 (2000).

8. J. A. Dewar, J. J. Gillogly, and M. L. Juncosa, "Non-Monotonicity, Chaos, and Combat Models," RAND Report R-3995-RC, 1991.

9. G. Rodriguez, C. R. Weisbin, and R. Easter, "A New Method to Compare the Performance of Humans, Robots, and Integrated Human-Robots Systems in $21^{\text {th }}$ Century Space Operations," JPL Publication 01-13, October 2001; see also: G. Rodriguez, C. R. Weisbin, and R. Easter, "A New Method for Human-Robots System Resiliency Evaluation,” JPL Publication 02-26, November 2002.

10. J.-L. Lions, “Optimal Control of Systems Governed by Partial Differential Equations, ” Springer Verlag, new York, 1971.

11. W. H. Fleming and R. W. Rishel, "Deterministic and Stochastic Optimal Control," Springer Verlag, New York, 1975.

12. E. Triantaphyllou, "Multi-criteria Decision Making Method: a Comparative Study,” Kluwer, Boston, 2000.

13. J. Geweke, ed., "Decision Making under Risk and Uncertainty: New Models and Empirical Findings,” Kluwer, Boston, 1992.

14. T. Biswas, “Decision Making under Uncertainty,” St. Martin’s Press, New York, 1997.

15. Y. Ben-Haim, "Information Gap: Decision Theory under Severe Uncertainty," Academic Press, San Diego, 2001 

ORNL/TM-2003/116

\section{INTERNAL DISTRIBUTION}

1. Jacob Barhen

2. C. W. Glover

3 - 7. S. Lenhart

8. J. A. Nichols

9 - 13. V. Protopopescu

14. D. B. Reister
15. R. T. Santoro

16. D. R. Tufano

17. Xiaoguang Zhang

18. Central Research Library

19 ORNL Laboratory Records - RC

20 - 21. ORNL Laboratory Records - OSTI

\section{EXTERNAL DISTRIBUTION}

22. Professor David R. Adams, Department of Mathematics, University of Kentucky, 937 Patterson Office Tower 0027, Lexington, KY 40506-0027

23. Professor Boris Belinskiy, Department of Mathematics, University of Tennessee at Chattanooga, 615 McCallie Avenue, Chattanooga, TN, 37403-2598

24. Professor Ethelbert Chukwu, Department of Mathematics, North Carolina State University, 242 Harrelson Hall, Box 8205, Raleigh, NC 27607-8205

25. Dr. Alberto Elfes, Strategic Systems Technology Program Office, Jet Propulsion Laboratory, 4800 Oak Grove Drive, MS-179-224, Pasadena, CA 91109-8099

26. Professor Renee Fister, 6C Faculty Hall, Department of Mathematics and Statistics, Murray State University, Murray, KY 42071

27. Dr. William Lincoln, Strategic Systems Technology Program Office, Jet Propulsion Laboratory, 4800 Oak Grove Drive, MS-179-224, Pasadena, CA 91109-8099

28. Dr. Guillermo Rodriguez, Strategic Systems Technology Program Office, Jet Propulsion Laboratory, 4800 Oak Grove Drive, MS-179-224, Pasadena, CA 91109-8099

29. Dr. Charles Weisbin, Deputy Program Manager, Strategic Systems Technology Program Office, Jet Propulsion Laboratory, 4800 Oak Grove Drive, MS-179-224, Pasadena, CA 91109-8099 